\title{
Dust production from collisions in extrasolar planetary systems
}

\section{The inner $\beta$ Pictoris disc}

\author{
P. Thébault ${ }^{1}$, J. C. Augereau ${ }^{2,3}$, and H. Beust ${ }^{4}$ \\ 1 Observatoire de Paris, Section de Meudon, 92195 Meudon Principal Cedex, France \\ 2 CEA Saclay, Centre de l'Orme des Merisiers, 91191 Gif-sur-Yvette Cedex, France \\ 3 Leiden Observatory, PO Box 9513, 2300 Leiden, The Netherlands \\ ${ }^{4}$ Laboratoire d'Astrophysique de l'Observatoire de Grenoble, Université J. Fourier, BP 53, 38041 Grenoble Cedex 9, France
}

Received 21 March 2003 / Accepted 19 June 2003

\begin{abstract}
Dust particles observed in extrasolar planetary discs originate from undetectable $\mathrm{km}$-sized bodies but this valuable information remains uninteresting if the theoretical link between grains and planetesimals is not properly known. We outline in this paper a numerical approach we developed in order to address this issue for the case of dust producing collisional cascades. The model is based on a particle-in-a-box method. We follow the size distribution of particles over eight orders of magnitude in radius taking into account fragmentation and cratering according to different prescriptions. Particular attention is paid to the smallest particles, close to the radiation pressure induced cut-off size $R_{\mathrm{pr}}$, which are placed on highly eccentric orbits by the stellar radiation pressure. We applied our model to the case of the inner $(<10 \mathrm{AU}) \beta$ Pictoris disc, in order to quantitatively derive the population of progenitors needed to produce the small amount of dust observed in this region $\left(\simeq 10^{22} \mathrm{~g}\right)$. Our simulations show that the collisional cascade from kilometre-sized bodies to grains significantly departs from the classical $\mathrm{d} N \propto R^{-3.5} \mathrm{~d} R$ power law: the smallest particles $\left(R \simeq R_{\mathrm{pr}}\right)$ are strongly depleted while an overabundance of grains with size $\sim 2 R_{\mathrm{pr}}$ and a drop of grains with size $\sim 100 R_{\mathrm{pr}}$ develop regardless of the disc's dynamical excitation, $R_{\mathrm{pr}}$ and initial surface density. However, the global dust to planetesimal mass ratio remains close to its $\mathrm{d} N \propto R^{-3.5} \mathrm{~d} R$ value. Our rigorous approach thus confirms the depletion in mass in the inner $\beta$ Pictoris disc initially inferred from questionable assumptions. We show moreover that collisions are a sufficient source of dust in the inner $\beta$ Pictoris disc. They are actually unavoidable even when considering the alternative scenario of dust production by slow evaporation of km-sized bodies. We obtain an upper limit of $\sim 0.1 M_{\oplus}$ for the total disc mass below $10 \mathrm{AU}$. This upper limit is not consistent with the independent mass estimate (at least $15 M_{\oplus}$ ) in the frame of the Falling Evaporating Bodies (FEB) scenario explaining the observed transient features activity. Furthermore, we show that the mass required to sustain the FEB activity implies a so important mass loss that the phenomena should naturally end in less than $1 \mathrm{Myr}$, namely in less than one twentieth the age of the star (at least $2 \times 10^{7}$ years). In conclusion, these results might help converge towards a coherent picture of the inner $\beta$ Pictoris system: a low-mass disc of collisional debris leftover after the possible formation of planetary embryos, a result which would be coherent with the estimated age of the system.
\end{abstract}

Key words. stars: planetary systems - stars: individual: $\beta$ Pictoris - stars: planetary systems: formation

\section{Introduction}

\subsection{The $\beta$ Pictoris system}

The dusty and gaseous $\beta$ Pictoris disc has been intensively studied since the first resolved image was obtained in 1984 (Smith $\&$ Terrile 1984). This system is particularly interesting since it is still one of the best examples of a possible young extrasolar planetary system. It should be stressed that considering the estimated age of the system, i.e. at least $2 \times 10^{7}$ years (Barrado y Navascués et al. 1999), $\beta$ Pictoris is no longer in its earliest formation stage and that if planetary accretion had to occur then it should already be finished.

Send offprint requests to: $\mathrm{P}$. Thébault, e-mail: philippe. thebault@obspm.fr
The $\beta$ Pictoris disc has been observed in various wavelengths and has a radial extent of at least $1500 \mathrm{AU}$ (Larwood \& Kalas 2001). Due to obvious observational constraints (10 AU at $\beta$ Pic's distance correspond to $\sim 0.5^{\prime \prime}$ ), it is mostly the outer part of the disc beyond $\sim 30$ AU that has been extensively mapped and studied with the highest spatial resolution (the reader might refer to Artymowicz 1997 for a complete review). The global picture is that of a relative central gap in the dust density, followed by a density peak around $100 \mathrm{AU}$ and a slow decrease outwards. The exact density profile remains relatively uncertain since it strongly depends on both the assumed size distribution for the dust population and the grain optical properties. As a consequence the total mass of dust is also relatively uncertain, but might be of the order of 0.3 to $0.5 M_{\oplus}$ 
(Li \& Greenberg 1998; Artymowicz 1997). Note that "dust" mass estimates always strongly depend on the upper size limit considered, and that observations do not constraint very well the abundance of the bigger dust particles where most of the mass is supposed to be. Observations show mostly the signature of micron-sized grains visible through scattered light (e.g. Kalas \& Jewitt 1995; Mouillet et al. 1997) and thermal emission (Lagage \& Pantin 1994). Millimetre-sized grains are detected by millimetre wavelength photometry (Chini et al. 1991; Zuckerman \& Becklin 1993) and by resolved imaging in the submillimeter domain but with a poor angular resolution (Holland et al. 1998).

Actually no direct information is available for all objects bigger than a few millimetres. Analytical estimates have shown that the observed dust cannot be primordial since its expected lifetime imposed by the rate of destructive mutual collisions is much shorter than the estimated age of the system (Artymowicz 1997; Lagrange et al. 2000). Thus dust must be constantly produced within the disc. Candidates for producing this dust are supposingly kilometer-sized planetesimals which may generate dust either by evaporation of volatiles (Li \& Greenberg 1998; Lecavelier 1998) or/and by collisional erosion (Artymowicz 1997; Mouillet et al. 1997; Augereau et al. 2001). In either case, the total mass of the disc must be dominated by these parent bodies. Artymowicz (1997) estimated that if a steady state collisional law in $\mathrm{d} N \propto R^{-3.5} \mathrm{~d} R$ (Dohnanyi 1969) holds from the smallest dust grains to the biggest planetesimals, then one might expect at least $140 M_{\oplus}$ of kilometre-sized objects. But, as noted by the author himself, such extrapolations remain very uncertain.

Scattered light observations have also revealed several more or less marked asymmetries in the outer disc (see Kalas \& Jewitt 1995, for a detailed presentation). Some of these asymmetries are believed to be due to the presence of an embedded planet. It is in particular the case of the slight warp in inclination $\left(\sim 3^{\circ}\right)$ of the disc's mid plane observed up to $80 \mathrm{AU}$. This warp has been successfully interpreted by the dynamical response of a planetesimal disc to the pull of a Jupiter-like object located at about $10 \mathrm{AU}$ from the star on a slightly inclined orbit (Mouillet et al. 1997). To extend this scenario to the dust disc moreover allows to reproduce large-scale vertical asymmetries up to about $500 \mathrm{AU}$ (Augereau et al. 2001). The planetary hypothesis is reinforced by new asymmetries evidenced at mid-IR wavelengths in the inner disc (Wahhaj et al. 2003).

\subsection{The inner disc}

Nevertheless, these indirect effects of an hypothetic planet are detected much further away from the star than the planet's actual location. As indicated in the previous section, there is a strong lack of data for the region within $10 \mathrm{AU}$ which is probably the most interesting area in terms of presence of planets and planet formation. Most of the informations on this region has been indirectly inferred by fitting the Spectral Energy Distribution (SED) in the near and middle infrared. There seems to be a general agreement on the fact that the inner part of the disc is significantly depleted in dust, though opinions strongly differ on the exact extension and intensity of this depletion (see Li \& Greenberg 1998, for a detailed discussion on this topic). To the present day, one of the most complete studies remains that of Li \& Greenberg (1998), taking into account a large set of parameters and especially realistic grain properties (porosity, size distributions, chemical compositions) based on observations, laboratory experiments and dust collection into space. This work claims that there is no more than $6 \times 10^{22} \mathrm{~g}$ of dust in the $r<40 \mathrm{AU}$ region, as compared to $6 \times 10^{25} \mathrm{~g}$ in the $[40,100] \mathrm{AU}$ area. The main problem is that such SED fits are strongly model dependent, and in particular that the dust surface density distribution cannot be uniquely determined because of its coupling to the grain size distribution and to the optical properties. Furthermore, the Li \& Greenberg (1998) fitting has been performed assuming that all dust is of pure comet-evaporation origin and that its size distribution fits in situ dust observations around the Halley comet. As will be discussed later on (Sect. 5), this assumption probably cannot hold for the inner Beta-Pic disc.

There is nevertheless one independent evidence for an inner dust depletion deduced from direct observations: Pantin et al. (1997) obtained resolved $12 \mu \mathrm{m}$ images of the $r<100 \mathrm{AU}$ region, with a resolution of $\sim 5 \mathrm{AU}$ after deconvolution. They concluded that there is a density drop of almost an order of magnitude in the innermost $r<10 \mathrm{AU}$ area, although a puzzling density peak seems to be observed at $5 \mathrm{AU}$. These authors inferred a total dust mass of $\sim 2.4 \times 10^{21} \mathrm{~g}$ for the $r<10 \mathrm{AU}$ area. Note that this estimate is also strongly model dependant, though it doesn't make any assumption concerning the mechanism producing the dust: the authors suppose a power law for the size distribution with a change of power law index at a given size (and thus 3 free parameters). The Pantin et al. (1997) mass estimate is significantly lower than the one that can be deduced from Li \& Greenberg (1998) for the same region, i.e. $\sim 2.5 \times 10^{22} \mathrm{~g}$, especially when taking into account the fact that the upper grain size limit of Li \& Greenberg (1998), $0.4 \mathrm{~mm}$, is smaller than the $1 \mathrm{~mm}$ limit of Pantin et al. (1997). Extrapolating the Li \& Greenberg (1998) estimate up to the $1 \mathrm{~mm}$ limit leads to a total dust mass of $\simeq 3.5 \times 10^{22} \mathrm{~g}$. But as mentioned before, all authors agree on one core assumption: there $i s$ a dust depletion in the inner $\beta$ Pictoris system.

\subsection{Parent bodies in the inner disc}

As pointed out by Artymowicz (1997) for instance, the dust is not primordial and must be constantly replenished by larger bodies. This author favours the scenario of dust production through collisional erosion rather than cometary evaporation, an assumption that we also believe to be the most reasonable one for the inner disc (see discussion in Sect. 5.3). It is then tempting to use this link as an indirect way to constrain the population of parent planetesimals, which is otherwise totally invisible to observations. The most simple way to do this is to suppose that a collisional equilibrium $\mathrm{d} N \propto R^{-3.5} \mathrm{~d} R$ power law applies from the parent bodies down to the observed micronsized grains. This is the usual assumption commonly made to easily derive parent bodies masses in extrasolar dust discs (e.g. 
Table 1. Summary of mass estimates for the inner 10 AU region, as derived from previous works.

\begin{tabular}{llcr}
\hline \hline Authors & Modeling frame & Size range & Mass \\
\hline \multirow{2}{*}{ Li \& Greenberg (1998) } & full SED fitting & {$[0.1 \mu \mathrm{m}, 0.4 \mathrm{~mm}]$} & $2.5 \times 10^{22} \mathrm{~g}$ \\
\cline { 2 - 4 } & when extrapolated with a $R^{-3.5}$ law & {$[10,50] \mathrm{km}$} & $2.5 \times 10^{-2} M_{\oplus}$ \\
\hline \multirow{2}{*}{ Pantin et al. (1997) } & $\begin{array}{l}\text { inversion of mid-IR } \\
\text { surface brightness profile }\end{array}$ & {$[0.1 \mu \mathrm{m}, 1 \mathrm{~mm}]$} & $2.4 \times 10^{21} \mathrm{~g}$ \\
\cline { 2 - 4 } & when extrapolated with a $R^{-3.5}$ law & {$[10,50] \mathrm{km}$} & $2 \times 10^{-3} M_{\oplus}$ \\
\hline Thébault \& Beust (2001) & FEB scenario & {$[10,50] \mathrm{km}$} & $15-50 M_{\oplus}$ \\
\hline
\end{tabular}

Augereau et al. 1999 for HR 7496 A). This would here lead to a mass of objects in the 10 to $50 \mathrm{~km}$ range comprised between $2 \times 10^{-3} M_{\oplus}$ (taking the Pantin et al. 1997, dust density) and $2.5 \times 10^{-2} M_{\oplus}$ (taking the Li \& Greenberg 1998, estimate). These values seem very low, especially compared to the $140 M_{\oplus}$ mass of planetesimals estimate for the whole system (Artymowicz 1997) which was in accordance with the picture of an "early Solar System". This tends to reinforce the image of a strong mass depletion in the inner disc. Note that the concurrent cometary evaporation scenario also gives a quantitative link between the observed dust and the source kilometre-sized comets (e.g. Eq. (2) of Lecavelier 1998), but this estimate does not constrain the number of non-evaporating objects.

There is nevertheless another way to get informations on the planetesimal population through the study of the so called Falling Evaporating Bodies (hereafter FEB) phenomenon. It is indeed believed that the evaporation of at least kilometer-sized bodies is responsible for the transient absorption features regularly observed in various spectral lines: CaII, MgII, FeII, etc. (e.g. Boggess et al. 1991; Vidal-Madjar et al. 1994; Beust et al. 1996). Several theoretical and numerical studies have shown that these FEB might be bodies located at the 3:1 and/or 4:1 resonances with a giant planet on a slightly eccentric orbit located around $10 \mathrm{AU}$. These objects are excited on high eccentricity $e$ orbits which allow them to pass sufficiently close to the star, i.e. less than $0.4 \mathrm{AU}$, for silicate to evaporate (see Beust \& Morbidelli 2000; Thébault \& Beust 2001, and references therein). Thébault \& Beust (2001) estimated that the number density of planetesimals required to fit the observed rate of absorption features would lead to a mass of $\simeq 15-50 M_{\oplus}$ objects in the 10 to $50 \mathrm{~km}$ range when assuming an equilibrium differential law in $R^{-3.5}$ in the inner $<10 \mathrm{AU}$ region. This very high estimate is close to the Artymowicz (1997) estimate for the whole disc and strongly exceeds, by at least a factor $10^{3}$, the above-mentioned much lower dust-mass-extrapolated estimations for the inner disc.

\subsection{The need for a numerical approach}

There is thus yet no coherent picture of the inner $\beta$-Pic system's structure, especially for the crucial link between the observed dust and unseen bigger parent bodies. The main reason for this is that deriving mass estimate from a simple power law from the micron to the kilometre might be strongly misleading.
A first argument is that a very small difference in the power law index leads to enormous differences when extrapolating it over such a wide size range. If $q$ is this index, then the mass ratio between 2 populations of sizes $R_{1}$ and $R_{2}$ reads $M_{1} / M_{2}=$ $\left(R_{1} / R_{2}\right)^{(q+4)}$. As an example, the incompatibility between the $15-50 M_{\oplus}$ FEB mass estimate and the $2 \times 10^{-3}-2.5 \times 10^{-2} M_{\oplus}$ extrapolated from the observed dust density might be solved when changing the $q$ index in the later extrapolation from -3.5 to -3.2 . But the single power law approach raises also other problems. Firstly, there is no reason why the upper size limit of the collisional cascade should be 10 or $50 \mathrm{~km}$. Extrapolating a $q=-3.5$ power law up to, say, $1000 \mathrm{~km}$ instead of $50 \mathrm{~km}$, would lead to a 4.5 times superior mass of large objects, thus reducing the magnitude of the inner mass depletion. But then, taking the same upper limit for the rest of disc (i.e. outside $10 \mathrm{AU})$ would increase the total mass of the system to unrealistically high values (more than $1000 M_{\oplus}$ ). In this case two different size distributions should hold for the inner and the outer systems.

Secondly, it is almost certain that a single -3.5 equilibrium power law cannot hold over such an extremely large size range. For such a power law to apply, all particles in the system should have reached mutual collisional equilibrium. This is perhaps not the case here, especially for the bigger bodies which, depending on their number density, might have low collision rates. Furthermore, such a power law is theoretically achieved only for an infinitely small lower size cutoff. As shown by Campo Bagatin et al. (1994), any finite size cutoff will give rise to wavy size distribution structures which can strongly differ from the theoretical -3.5 slope. The reason for such size distribution waves is simple: the smallest particles will be overabundant since they have no smaller bodies to destroy them. This over-abundance will give rise to an under-abundance for all bigger bodies that might be collisionally fragmented by these minimum-sized objects. This will in turn lead to an overabundance of bigger bodies, and so on. This point is of great importance here since there is an obvious size cutoff for our system, i.e. the smallest grains that are not blown away by the star's radiation pressure and which are typically micron-sized. Apart from this cutoff effect, the smallest grains are also expected to have a very peculiar behaviour: even if radiation pressure is not able to remove them, it should nevertheless place them on highly eccentric orbits, thus augmenting their impact velocities and shattering power, but at the same time reducing 
their density in the inner region since they will spend most of their orbits very far away from the star. The physical link between dust and planetesimals is thus a complex one, that cannot be handled by simple analytical power laws.

We propose here to address these problems by performing accurate numerical simulations. A statistical particle-in-a-box code is used to study the mutually coupled collisional evolution of a swarm of objects ranging in size from large planetesimals down to the smallest micron-sized grains. The code is similar to the ones developed for asteroid populations studies but stretches down to very small dust particles and takes into account the peculiar dynamical evolution of micron-sized grains submitted to the star's radiation pressure. We detail in Sects. 2 and 3 the numerical approach we use to derive a realistic grain size distribution at a distance of a few AU resulting from a collisional cascade in the radiative environment of $\beta$ Pictoris. We explore the impact of the free parameters, along with the two extreme surface densities independently deduced from dust and gas observations of $\beta$ Pictoris, on the final size distribution after $10 \mathrm{Myr}$ (Sect. 4). We then discuss in Sect. 5 the implications of our approach and show how it helps to go towards a coherent view of the inner $\beta$ Pictoris disc (Sect. 6).

\section{Numerical procedure}

We will here follow the classical particle in a box approach used by models studying the asteroid belt size distribution (e.g. Petit \& Farinella 1993). We consider a typical annulus of material in the inner disc, of radius $1 \mathrm{AU}$ and located at $5 \mathrm{AU}$ from the star. The system is divided into $n$ boxes accounting for each particle size within the annulus. The size increment between two adjacent bins is $2^{1 / 3}$. At each time step the evolution of the number $\mathrm{d} N_{k}$ of bodies of size $R_{k}$ is given by

$\mathrm{d} N_{k}=\sum_{i, j=1}^{n} n_{i, j, k} p_{i, j} N_{i} N_{j} \mathrm{~d} t-\sum_{l=1}^{n} \gamma_{l, k} p_{l, k} N_{l} N_{k} \mathrm{~d} t$

where $n_{i, j, k}$ is the number of fragments injected into the $k$ bin by an impact between 2 bodies in the $i$ and $j$ bins and $p_{i, j}$ the impact rate for a pair belonging to the same two bins. The last term of the equation accounts for the loss of $k$ objects due to destructive collisions $\left(\gamma_{l, k}=1\right.$ for a catastrophic fragmentation impact and $0<\gamma_{l, k}<1$ (satisfying the mass conservation condition) for a cratering event). The temporal evolution of $N_{k}$ is then computed using a first order eulerian code with a variable time step. One key information needed for estimating $n_{i, j, k}, \gamma_{l, k}$ and $p_{i, j}$ is the dynamical state of the system, which can be parameterised by the average impact velocities $\langle\mathrm{d} v\rangle$.

\subsection{Dynamical state of the system}

As stated in the previous section, there is yet no clear picture of the system we intend to study. We have in particular no precise idea of the dynamical state of the inner disc. There is nevertheless some indirect evidence of the dynamical state in the outer parts, given by the observed thickness of the disc. The disc aspect ratio in the $100 \mathrm{AU}$ region is believed to be $\simeq 0.1$ (Augereau et al. 2001). Thus, a first order approximation of the average inclination of the observed dust particles would be half this value, i.e. $\simeq 0.05 \mathrm{rd}$. However, this value only gives very partial information, and this for several reasons:

1. It is not straightforward to extrapolate it to the inner regions of the disc, where the dynamical conditions could be completely different. Indeed Kalas \& Jewitt (1995) seem to observe a significant departure from constant disc opening for $r<60 \mathrm{AU}$, but such determinations should be taken with great care, since measures of the disc's thickness become very uncertain for these inner regions.

2. This value holds for the observed micron to millimetresized grains population. It is not at all certain that bigger parent bodies have the same inclinations.

3. The dynamical state of the system depends on the inclination and eccentricity distributions. The $\langle e\rangle$ value cannot be directly deduced from $\langle i\rangle$, at least for the micron-sized population, where orbits might strongly depart from the equilibrium $\langle i\rangle=\langle e\rangle / 2$ equipartition relation (points 2 and 3 will be discussed in more details in Sect. 3).

We will thus take the $\langle e\rangle$ and $\langle i\rangle$ values of the parent bodies in the considered $r<10 \mathrm{AU}$ region as free parameters (see Sect. 4.3), but we will nevertheless refer to the $\langle i\rangle=0.05=$ $\langle e\rangle / 2$ case as our "nominal" case. Note that our simulations do not directly use the $\langle e\rangle$ and $\langle i\rangle$ parameters but the average relative velocity parameter $\langle\mathrm{d} v\rangle$ given by Lissauer \& Stewart (1993):

$\langle\mathrm{d} v\rangle=\left(\frac{5}{4}\left\langle e^{2}\right\rangle+\left\langle i^{2}\right\rangle\right)^{1 / 2}\left\langle v_{\mathrm{kep}}\right\rangle$

where $\left\langle v_{\mathrm{kep}}\right\rangle$ is the average Keplerian velocity of the bodies. Furthermore, we will assume the same $\langle e\rangle=\left\langle e_{0}\right\rangle,\langle i\rangle=\left\langle i_{0}\right\rangle$ and thus $\left\langle\mathrm{d} v_{i, j}\right\rangle=\left\langle\mathrm{d} v_{0}\right\rangle$ for all particles, with the important exception of the micron-sized grains which are significantly affected by the star's radiation pressure (see Sect. 3 for more details). We will also make the simplifying assumption that our particle in a box system is not dynamically evolving, so that $\langle\mathrm{d} v\rangle$ remains constant throughout the run.

\subsection{Density of objects}

As described in Sects. 1.2 and 1.3, there are two independent estimates of the density of bodies in the inner disc: 1) a dust mass (all bodies smaller than $1 \mathrm{~mm}$ ) of $2.4 \times 10^{21}-3.5 \times 10^{22} \mathrm{~g}$ derived from fits of the observed SED 2) a mass of 15-50 $M_{\oplus}$ of planetesimals in the $10-50 \mathrm{~km}$ range required to sustain the FEB activity. As previously discussed, these 2 estimates appear totally incompatible when assuming an equilibrium differential $R^{-3.5}$ size distribution throughout the system, since in this case the mass of planetesimals extrapolated from the dust estimate is only $2 \times 10^{-3}-2.5 \times 10^{-2} M_{\oplus}$. In order to check how strong an incompatibility there really is, or if there is any incompatibility at all, we will consider two extreme initial discs (see Sects. 4.1 and 4.2):

- An initial $R^{-3.5}$ distribution extending from $R_{\min }=2^{-2 / 3} R_{\mathrm{pr}}$ (i.e. 2 boxes under the $R_{\mathrm{pr}}$ ejection size, see Sect. 3) up to $R_{\max }=50 \mathrm{~km}$ which is compatible with observed dust 
mass estimates. We chose to take an initial dust mass of $2 \times 10^{22} \mathrm{~g}$ in the whole inner disc (i.e. $\simeq 2 \times 10^{21} \mathrm{~g}$ in the considered $1 \mathrm{AU}$ annulus around $5 \mathrm{AU}$ ), an intermediate value between the Pantin et al. (1997) and Li \& Greenberg (1998) estimates. This leads to a total initial mass of $1.8 \times$ $10^{25} \mathrm{~g}$ for the whole system. This initial density distribution will be taken for our "nominal" case;

- An initial $R^{-3.5}$ distribution extending from $R_{\min }=2^{-2 / 3} R_{\mathrm{pr}}$ up to $R_{\max }=50 \mathrm{~km}$ and compatible with an average FEB estimate of $\simeq 25 M_{\oplus}$ of $10-50 \mathrm{~km}$-sized objects in the $1-$ $10 \mathrm{AU}$ region (i.e. $\simeq 2.5 M_{\oplus}$ in the considered annulus), which leads to a total initial mass of $3.5 \times 10^{28} \mathrm{~g}$.

Regardless of the initial density distribution, the number of size boxes considered is always the same for a given $R_{\mathrm{pr}}$, ranging from $R_{\min }=2^{-2 / 3} R_{\mathrm{pr}}$ to $R_{\max }=50 \mathrm{~km}$, with two adjacent boxes separated by a factor $2^{1 / 3}$ in size, thus leading to a total number of 103 boxes for the "nominal" case where $R_{\mathrm{pr}}=5 \times 10^{-4} \mathrm{~cm}$ (see Sect. 3).

\subsection{Threshold specific energy}

The core of such a code is the prescription giving $n_{i, j, k}$ for a given $\langle\mathrm{d} v\rangle$. Basically, impacts can be divided into two categories: catastrophic fragmentation and cratering, depending on the value of the impacting energy as compared to $Q_{*}$, the threshold specific energy of the bodies, which represents their resistance to shattering and is deduced from laboratory experiences and analytical considerations. $Q_{*}$ is by definition the value of the specific energy $Q$ (the ratio of the projectile kinetic energy to the target mass) when the mass of the largest remaining fragment $M_{l f(i)}$ is equal to $0.5 M_{i}$.

The problem is that estimations of $Q_{*}$ do strongly differ from one author to another (see Fig. 8 of Benz \& Asphaug 1999, for an overview). Basically, all authors agree on one core assumption, i.e. the response of solid bodies to impacts is divided in two distinct regimes: the strength regime for small bodies, where the object's resistance decreases with size, and the gravity regime for larger objects where resistance increases with size because of the object's self-gravity (e.g. Housen \& Holsapple 1990). Nevertheless, the slopes and turn over size from one regime to another are still a great subject of debate. We will here consider separately two different $Q_{*}$ prescriptions (see Sect. 4.5):

- the global strength + gravity regime law given in Eq. (6) of Benz \& Asphaug (1999) (nominal case),

- the Housen \& Holsapple (1990) law for the strength regime completed by the Holsapple (1994) law for the gravity regime.

Our code calculates for every target-impactor couple $(i, j)$ the corresponding value of $Q_{*(i, j)}$. Let us term $F_{l f(i, j)}$ the ratio $M_{l f(i)} / M_{i}$. From the value of $Q_{*}, F_{f l(i, j)}$ can be inferred through the empirical relation (Fujiwara et al. 1977):

$F_{l f(i, j)}=0.5\left(\frac{Q_{*} M_{i}}{E_{\mathrm{rel}}}\right)^{1.24}$

where $E_{\mathrm{rel}}$ is the relative kinetic energy of the system given by $E_{\text {rel }}=M_{i} M_{j} \mathrm{~d} v^{2} / 2\left(M_{i}+M_{j}\right)$. Note that this relation is valid only for head-on impacts and has to be corrected by taking into account its value averaged over all impacts angles. We will here follow Petit \& Farinella (1993) and take the average value:

$\overline{F_{l f(i, j)}}=3 F_{f l(i, j)}^{2 / 3}-2 F_{f l(i, j)}$.

Thus, Eq. (3) has to be corrected by a numerical factor $x_{\mathrm{cr}}=$ $4^{-1 / 1.24}=0.327$, since $\overline{F_{l f(i, j)}}=1 / 2$ for $F_{l f(i, j)}=1 / 8$.

\subsection{Fragmentation}

Catastrophic fragmentation occurs by definition when $F_{l f(i, j)}$ is less than 0.5 . If we suppose that the produced fragment size distribution follows a single-exponent power law $\mathrm{d} N=C R^{q} \mathrm{~d} R$, then there is a unique set of values for $q$ and $C$ derived from the value of $F_{l f(i, j)}$ and the mass conservation condition. As pointed out in several previous studies, this single power law specification is the easiest to handle in models but it is a strong oversimplification. It gives rise to several problems, in particular the possibility to get so-called "supercatastrophic" impacts where $q<-4$, for which there is a divergence of the total mass when taking infinitely small lower cutoff. As noted by Tanga et al. (1999) “...values beyond -3 for the exponent of the cumulative size distribution cannot hold down to very small sizes, because this would lead to unreasonably large reconstructed masses. For this reason it is clear that, at some value of the size, the distributions are expected to have a definite change of slope". Note that this change of slope between the small and large fragments domain is also supported by experimental experiments (Davis \& Ryan 1990). This problem is particularly crucial for the present study since our size cutoff is extremely small (see below).

As a consequence, we will here adopt 2 different power laws of index $q_{1}$ and $q_{2}$, each holding for a different mass range and always taken such as the small mass index $q_{2}$ is smaller than $q_{1}$. The main problem is to determine where the change of slope occurs and what the difference in slope is. We shall remain careful and keep the slope changing size $R_{\mathrm{s}(i)}$ as well as the ratio $q_{1 i} / q_{2 i}$ as free parameters that will be explored in the runs (see Sect. 4.6). Note that once $R_{\mathrm{S}}$ and $q_{1 i} / q_{2 i}$ are given, the values $q_{1 i}$ and $q_{2 i}$ for the fragments produced on a target $i$ by an impactor $j$ are uniquely determined through the set of relations:

$M_{1 i}=\left[\frac{b_{1 i} M_{l f(i)}^{b_{1 i}}}{\left(1-b_{1 i}\right)}\left(M_{l f(i)}^{\left(1-b_{1 i}\right)}-M_{\mathrm{s}(i)}^{\left(1-b_{1 i}\right)}\right)+M_{l f(i)}\right]$

$C_{1 i}=3 b_{1 i} R_{l f(i)}^{3 b_{1 i}}$

$C_{2 i}=\frac{3 b_{1 i} R_{l f(i)}^{3 b_{1 i}}}{R_{\mathrm{s}(i)}^{3\left(b_{1 i}-b_{2 i}\right)}}$

$\frac{C_{2 i}}{\left(3-3 b_{2 i}\right)} R_{\mathrm{s}(i)}^{-3 b_{2 i}}=\frac{M_{i}-M_{1 i}}{M_{\mathrm{s}}(i)}$

where $b_{1 i}=-\frac{1}{3}\left(q_{1 i}+1\right)$ and $b_{2 i}=-\frac{1}{3}\left(q_{2 i}+1\right), M_{\mathrm{S}(i)}$ is the mass of an object of size $R_{\mathrm{s}(i)}, M_{1 i}$ is the total mass of fragments produced in the domain where the $\mathrm{d} N=C_{1 i} R^{q 1 i} \mathrm{~d} R$ law applies, 
i.e. between the size of the slope transition $R_{\mathrm{S}(i)}$ and the size of the largest fragment $R_{l f(i)}$, and $C_{1 i}$ and $C_{2 i}$ are the coefficients for the two $\mathrm{d} N=C_{1 i} R^{q 1 i} \mathrm{~d} R$ and $\mathrm{d} N=C_{2 i} R^{q 2 i} \mathrm{~d} R$ power laws. This set of equation is solved numerically for each $(i, j)$ couple.

\subsection{Cratering}

For the cratering case $\left(F_{l f}>0.5\right)$, we will take the simplified prescription of Petit \& Farinella (1993), where a fixed power law index $q_{\mathrm{c}}=-3.4$ is considered. The total mass of craterized mass is given by

$M_{\mathrm{cr}}=\alpha E_{\mathrm{rel}}$ for $E_{\mathrm{rel}} \leq \frac{M_{i}}{100 \alpha}$,

$M_{\mathrm{cr}}=\frac{9 x_{\mathrm{cr}} \alpha}{100 Q_{*} \alpha-x_{\mathrm{cr}}} E_{\mathrm{rel}}+\frac{M_{i}}{10} \frac{x_{\mathrm{cr}}-10 Q_{*} \alpha}{x_{\mathrm{cr}}-100 S_{*} \alpha}$

for: $E_{\mathrm{rel}}>\frac{M_{i}}{100 \alpha}$

where $\alpha$ is the crater excavation coefficient which depends on the material properties. We will explore values of $\alpha$ (Sect. 4.6) ranging from $10^{-9}$ to $4 \times 10^{-8} \mathrm{~s}^{2} \mathrm{~cm}^{-2}$, the extreme values corresponding to "hard" and "soft" material respectively (see Petit \& Farinella 1993, and references therein), and take $10^{-8} \mathrm{~s}^{2} \mathrm{~cm}^{-2}$ as our "nominal" value. The mass of the largest fragment produced by the impact is then equal to $F_{l_{\mathrm{cr}}} M_{\mathrm{cr}}$, where $F_{l_{\mathrm{cr}}}=$ $1+\frac{1}{3}\left(q_{\mathrm{c}}+1\right)$

\subsection{Fragment reaccumulation}

The fraction of fragmented material reaccumulated onto the parent bodies is the result of the competing ejectas' kinetic energy and the parent bodies' gravitational potential. We will make the simplified assumption that all fragments produced after an $(i, j)$ impact have the same velocity distribution (Stern \& Colwell 1997):

$v_{f r}=\left(2 \frac{f_{\mathrm{ke} E_{\mathrm{rel}}(i, j)}}{M_{i}}\right)^{1 / 2}$

where $f_{\mathrm{ke}}$ is the fraction of kinetic energy that is not dissipated after an impact. We will make here the classical assumption that $f_{\mathrm{ke}}=0.1$ for high velocity impacts (Fujiwara et al. 1989). The mass fraction of fragment material that escapes the target+impactor system is given by (Stern \& Colwell 1997):

$f_{\mathrm{esc}}=0.5\left(\frac{v_{\mathrm{esc}}}{v_{\mathrm{fr}}}\right)^{-1.5}$

where $v_{\text {esc }}$ is the escape velocity of the colliding bodies system.

\section{The specific behaviour of the micron-sized population}

The main challenge of this simulation is that we would like to study the collisional correlation between objects ranging from the micron-sized to the kilometre-sized domain, i.e. separated by 8 orders of magnitude in size. Our lower cutoff is indeed the "real" physical cutoff $R_{\mathrm{pr}}$ imposed by the effect of the star's radiation pressure. Radiation pressure also strongly affects particles bigger than $R_{\mathrm{pr}}$, but still in the same size range, by placing them on highly eccentric orbits. These eccentricities depend on the ratio $\beta$ between the radiation pressure force $F_{\mathrm{pr}}$ and the gravitational force $F_{\text {grav }}$. For a particle produced by a parent body on a $\left(a_{0}, e_{0}\right)$ orbit at a distance $r_{0}$ from the star, one gets:

$a_{k}=\frac{1-\beta}{1-2 a_{0} \beta / r_{0}} a_{0}$

$e_{k}=\left(1-\frac{\left(1-2 a_{0} \beta / r_{0}\right)\left(1-e_{0}^{2}\right)}{(1-\beta)^{2}}\right)^{1 / 2}$

where $a_{k}$ and $e_{k}$ are the produced grain's semi-major axis and eccentricity and $a_{0}$ and $e_{0}$ the semi-major axis and eccentricity of the parent body. From Eq. (14) and with the assumption that the planetesimals releasing dust particles by collisions are mostly on circular orbits $\left(e_{0} \simeq 0, a_{0} \simeq r_{0}\right)$, then grains with $\beta \geq 0.5$ are ejected from the system on hyperbolic orbits. The cutoff size $R_{\mathrm{pr}}$ is by definition the size for which $\beta=0.5$ and grains with sizes $R$ larger than $R_{\mathrm{pr}}$ are related to $\beta$ through the relation $\beta=0.5\left(R_{\mathrm{pr}} / R\right)$. The blow-out size $R_{\mathrm{pr}}$ depends on the stellar spectra and on grains optical properties. For $\beta$ Pictoris we use a low-resolution A5V spectra derived from Kurucz stellar models and we adopt the chemical grain composition proposed by Li \& Greenberg (1998) (we refer to the latter paper and to Augereau et al. 1999, for a full discussion on chemical and optical properties of the grains assumed here). Bare compact silicate ("Si") grains in the surroundings of $\beta$ Pictoris and smaller than $R_{\mathrm{pr} \text {, compact }} \simeq 3.5 \mu \mathrm{m}$ have $\beta \geq 0.5$. The same grains but coated by an organic refractory ("or") mantle in a Si:or volume ratio of 1:2 as proposed by Li \& Greenberg (1998) are ejected from the system on unbound orbits if they are smaller than $R_{\mathrm{pr} \text {, compact }} \simeq 2.5 \mu \mathrm{m}$. Actually the main uncertainty on $R_{\mathrm{pr}}$ relies on the grain porosity $P$. The porosity affects the optical properties of the grains and consequently $F_{\text {rad }}$. But actually the $\beta$ ratio more dramatically depends on $P$ through the grain density in $F_{\text {grav }}$ especially for large porosities. The density of porous grains is related to the density of the same but compact grain by the simple relation: $\rho_{\text {porous }}=(1-P) \rho_{\text {compact }}$ which implies for large porosities: $R_{\mathrm{pr} \text {, porous }} \simeq(1-P)^{-1} R_{\mathrm{pr} \text {, compact }}$. From SED fitting, Li \& Greenberg (1998) constrained $P$ in the narrow range $[0.95,0.975]$. But these values are obtained when assuming that all grains are of cometary origin, an assumption that we believe might not hold for inner $\beta$ Pictoris disc (see the more complete discussion in Sect. 5.3). In the present paper we keep $P$ has a free parameter with $R_{\mathrm{pr}}=5 \mu \mathrm{m}$ (i.e. $P \simeq 0.5$ ) taken as our reference nominal case (see Sect. 4.4).

The radiation pressure induced eccentricity expressed by Eq. (14) is significant, say $\geq 0.1$, for all particles comprised between $R_{\mathrm{pr}}$ and $\sim 5 R_{\mathrm{pr}}$. Thus all objects in this size range will have orbital characteristics that depart from the general average values defined in Sect. 2.1. This will significantly affect the collision rates and physical outcomes for impacts involving these small grains. For these impacts, Eq. (2) is no longer valid in its simple form and $\left\langle\mathrm{d} v_{i, j}\right\rangle$ will be numerically estimated. To do this, we use a simplified version of a deterministic collisional model (Thébault et al. 2002, and references therein) 
Table 2. Numerical estimate of $\left\langle f_{i(k)}\right\rangle$ in the 5 AU region, for a swarm of grains produced in the whole 1-10 AU region, as a function of $\beta_{k}$ (see text for details). Note that for low $\beta, e_{k}$ might become lower than the average eccentricity for the parent bodies in the disc (see Sect. 2.1). In such a case, the radiation pressure effect is neglected for all $e_{k}<$ $\left\langle e_{0}\right\rangle$ and the values of $\left\langle f_{i(k)}\right\rangle$ rescaled so that $\left\langle f_{i(k)}\right\rangle=1$ for $e_{k}=\left\langle e_{0}\right\rangle$.

\begin{tabular}{ccc}
\hline \hline$\beta_{k}$ & $e_{k}$ & $\left\langle f_{i(k)}\right\rangle$ \\
\hline 0.49 & 0.96 & 0.038 \\
0.39 & 0.63 & 0.344 \\
0.31 & 0.45 & 0.521 \\
0.24 & 0.32 & 0.665 \\
0.19 & 0.24 & 0.753 \\
0.15 & 0.18 & 0.812 \\
0.12 & 0.14 & 0.849 \\
0.10 & 0.11 & 0.887 \\
0.08 & 0.09 & 0.930 \\
\hline
\end{tabular}

to derive average $\langle\mathrm{d} v\rangle$ between a population of targets having the nominal orbital characteristics as defined in Sect. 2.1 and a population of impactors with a given $\beta_{k}$ (i.e. $a_{k}$ and $e_{k}$ ), all randomly distributed within the 1-10 AU region.

Another major consequence of these radiation-induced high $e_{k}$ is that small grains will spend a significant fraction of their orbits outside the inner disc. Thus, at a given moment, only a fraction $f_{i(k)} N_{k}$ of these bodies will actually be present in the considered system. These $\left\langle f_{i(k)}\right\rangle$ are numerically estimated with a simple code randomly spreading 10000 test particles of a given $\beta_{k}$ uniformly produced in the $1-10 \mathrm{AU}$ region (Table 2).

\subsection{Timescale}

It is important to note that these $\left\langle f_{i(k)}\right\rangle$ values are not reached instantaneously: small grains produced after an impact need time to reach the remote aphelion of their high $a$ and high $e$ orbits. If $\mathrm{d} t_{e j(k)}$ is the typical time needed for a small grain produced in the inner disc to reach $r=10 \mathrm{AU}$ when placed on a high $a_{k}$ and $e_{k}$ orbit, then during a time step $\mathrm{d} t_{1}$, the fraction of produced $k$ grains that leaves the system will be approximated through the simplified relation:

$f_{i(k)}^{\prime}=\left(1-f_{i(k)}\right) \cdot\left(1-\mathrm{e}^{-\frac{\mathrm{d} t_{1}}{\mathrm{~d} t_{e j(k)}}}\right)$.

Bodies that did not leave the system during $\mathrm{d} t_{1}$ are then added to a "bodies on their way to leave" subdivision of the $k$ population, that will in turn decrease by a $\left(1-f_{i(k)}\right) \cdot\left(1-\mathrm{e}^{-\frac{\mathrm{d} t_{2}}{\mathrm{~d} e_{e j(k)}}}\right)$ fraction at the next time step $\mathrm{d} t_{2}$, etc.

\subsection{Collisional destruction outside the inner disc}

Another possible effect affecting the smallest high $\beta$ particles is that a fraction of them might be destroyed by collisions outside the considered inner disc, since they spend an important fraction of their orbit close to their apoastron which might lie beyond $10 \mathrm{AU}$. These collisions would prevent them from re-entering the inner system. Such collisions are by definition not modeled by the collisional evolution Eq. (1), and this could lead to an overestimation of the density of these bodies.
Taking into account this apoastron-collisions removal effect requires one to make physical assumptions about the external parts of the disc and would add several badly constrained free parameters to our already large set of variables, in particular the rates and timescales for these collisional destructions as a function of $\beta$. We nevertheless tried to investigate the possible importance of this effect by performing test runs where we artificially introduced a new parameter $f_{c l(k)}$, standing for the fraction of $k$ bodies destroyed by collisions in the $r>10 \mathrm{AU}$ region and the corresponding destruction time scale $\mathrm{d} t_{c l(k)}$. The induced removal of $k$ bodies is then treated the same way as in Eq. (15). $f_{c l(k)}$ might be taken equal to the fraction of $\beta_{k}$ grains produced within the inner 1-10 AU disc which have their periastron outside $10 \mathrm{AU}$. The dependency of $\mathrm{d} t_{c l(k)}$ with $\beta_{k}$ is more difficult to establish, and several values will be explored.

As will be shown in Sect. 4.7, the obtained results do not significantly depart from our "nominal" case. As a consequence, and for sake of clarity, we chose to neglect, in a first approximation, this apoastron-collision effect.

\subsection{Particles with $\beta>0.5$}

Even if these particles' ultimate fate is to leave the system, their ejection takes also a certain amount of time and numerous very small grains, in this transition phase towards ejection, might be present in the system and thus collisionaly interacting with other objects. As a consequence, our runs will be performed with 2 bins below the limiting $\beta=0.5$ size. The fraction of $\beta>0.5$ bodies that do not leave the system after an impact is computed the same way as in Eq. (15), by numerically estimating $\mathrm{d} t_{e j(k)}$ and setting $f_{i(k)}=0$.

\section{Results}

We present here the results obtained for several runs exploring all important parameters the system's collisional evolution depends on. As previously mentioned, we define as our "nominal" case the one defined in Sect. 2.1. Initial conditions for this reference case are summarized in Table 3. For sake of clarity, all other parameters are separately explored in individual runs, even though some parameters should in principle not be independently explored, like in particular the value of $R_{\mathrm{pr}}$ (i.e. the grains' porosity) and the fragmentation and/or cratering prescriptions. All runs are carried out until $t_{\text {final }}=10^{7}$ years, i.e. approximately one third of the minimum age of the system (Barrado y Navascués et al. 1999).

\subsection{Nominal case}

Figure 1 shows clearly how the system quickly departs from the initial $R^{-3.5}$ distribution. A wavy structure rapidly appears because of the minimum size cut-off, in accordance with Campo Bagatin et al. (1994). This structure is building up progressively, starting from the lowest sizes and expanding towards the bigger objects bins. A quasi steady-state is reached after $\sim 10^{6}$ years and no significant further evolution of the system is observed in the next $9 \times 10^{6}$ years, except for a slow 
Table 3. Initial parameters for the nominal reference run (see text for details).

\begin{tabular}{ll}
\hline \hline Minimun size bin & $3.15 \times 10^{-4} \mathrm{~cm}$ \\
Maximum size bin & $5.4 \times 10^{6} \mathrm{~cm}$ \\
$R_{\mathrm{pr}}(\beta=0.5)$ & $5 \times 10^{-4} \mathrm{~cm}$ \\
$\left\langle e_{0}\right\rangle$ & 0.1 \\
$\left\langle i_{0}\right\rangle$ & 0.05 \\
$Q_{*}$ law & Benz \& Asphaug (1999) \\
$b_{1} / b_{2}$ & 1.5 \\
$R_{\mathrm{s}(i)}$ & $R_{(i)} / 2 \times 10^{5}$ \\
Excavation coefficient $\alpha$ & $10^{-8} \mathrm{~s}^{2} \mathrm{~cm}^{-2}$ \\
Initial density & $\mathrm{d} N \propto R^{-3.5} \mathrm{~d} R$ distribution \\
& with $M_{\text {dust }}=2 \times 10^{21} \mathrm{~g}$ in the annulus \\
& $\left(M_{\text {dust }}=2 \times 10^{22} \mathrm{~g}\right.$ in the whole inner \\
& disc $)$ \\
\hline
\end{tabular}

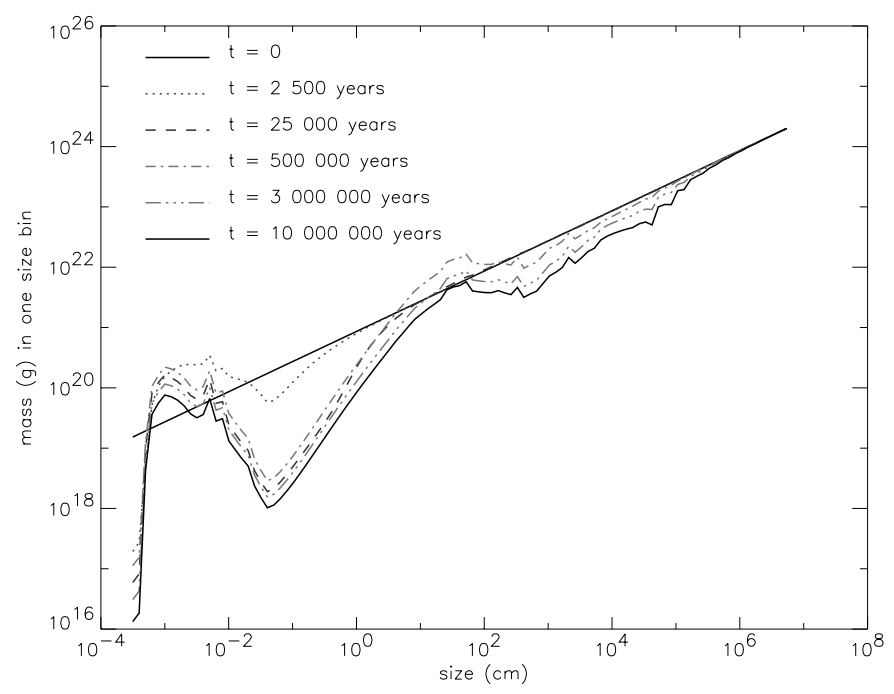

Fig. 1. Size distribution for the low-mass nominal system (see Table 3) at 5 different epochs. Note that the $y$-axis displays the mass contained in one size bin, which is a correct way of displaying the mass distribution since all size bins are equally spaced in a logarithmic scale. This plot is more "visual" than a more classical $\mathrm{d} N(R)$ one, since it can be directly interpreted in terms of mass contribution (and mass loss or mass increase) of each size range to the total mass.

decrease of the system's total mass. As could be logically expected, the wavy structure is the most significant in the small size domain. There is in particular a strong mass depletion, of a factor $\simeq 40$, in the $10^{-2}-1 \mathrm{~cm}$ range, with the lowest density point around $R \sim 0.1 \mathrm{~cm}$. This depletion has 2 distinct causes: 1) the overabundance of very small particles due to the size cutoff. Note however that this overabundance, though still present, is significantly damped or even erased for the smallest particles (close to $R_{\mathrm{pr}}$ ), because these bodies spend a significant fraction of their orbits outside the inner disc (see the $f_{i(k)}$ parameter in Table 2). 2) The high $\langle\mathrm{d} v\rangle$ values for impacts involving particles close to $R_{\mathrm{pr}}$, which are on highly eccentric orbits.

Another important result is that the total mass loss of the system over $10^{7}$ years remains relatively limited, i.e. less than $12 \%$ (cf. Fig. 2). Furthermore, the ratio $M_{\text {dust }} / M_{\text {planetesimals }}$, after large initial variations, progressively converges towards a value which is $\simeq 1 / 3$ of the $\mathrm{d} N=C R^{-3.5} \mathrm{~d} R$ power law value. This

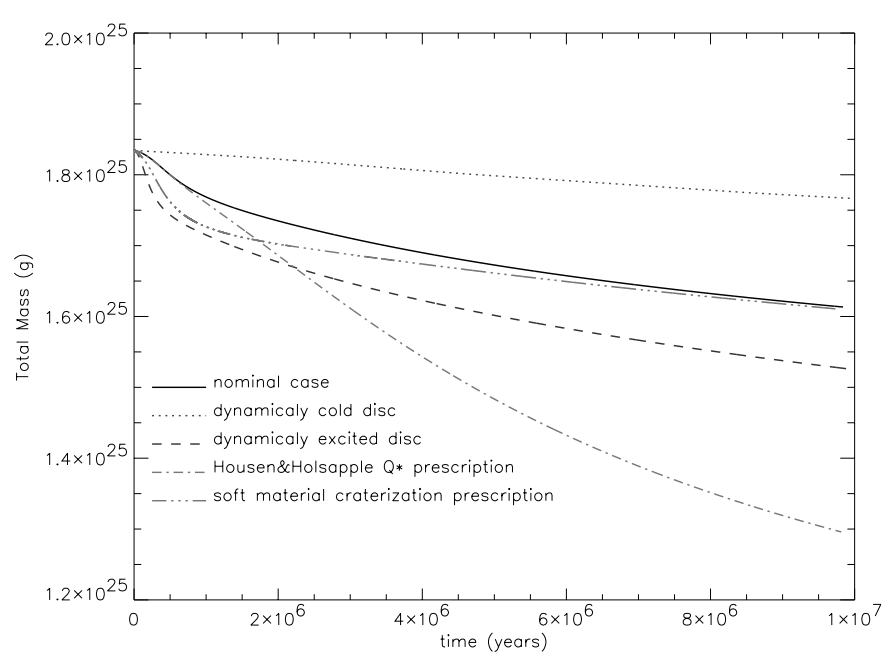

Fig. 2. Temporal evolution of the system's total mass for different cases.

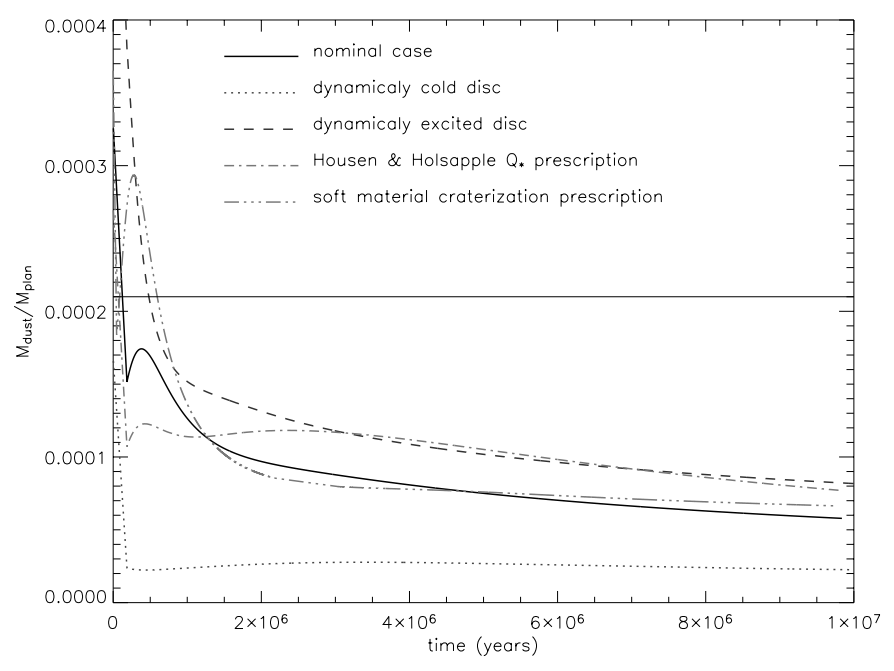

Fig. 3. Temporal evolution of the ratio $M_{\text {dust }} / M_{\text {planetesimals }}$ for different cases, where $M_{\text {dust }}$ is the total mass of all objects smaller than $1 \mathrm{~mm}$ and $M_{\text {planetesimals }}$ is the mass of all objects bigger than $10 \mathrm{~km}$. The horizontal line gives the initial value corresponding to an academic $\mathrm{d} N=C R^{-3.5} \mathrm{~d} R$ size distribution.

is mostly due to a decrease of $M_{\text {dust }}$, with $M_{\text {planetesimals being }}$ almost constant.

\subsection{Massive disc}

The massive-disc case turns out to be significantly different (Fig. 4). Although a quasi steady-state is here also rapidly reached and has a profile similar to that of the nominal case, this steady-state is obtained for a much higher density. This leads to much faster mass loss than in the previous case, exceeding one order of magnitude at the end of the simulation (Fig. 5), since mass loss in a given collisional system increases with the square of the system's density. We discuss the implications of these results on the FEB phenomena in Sect. 5.1. 


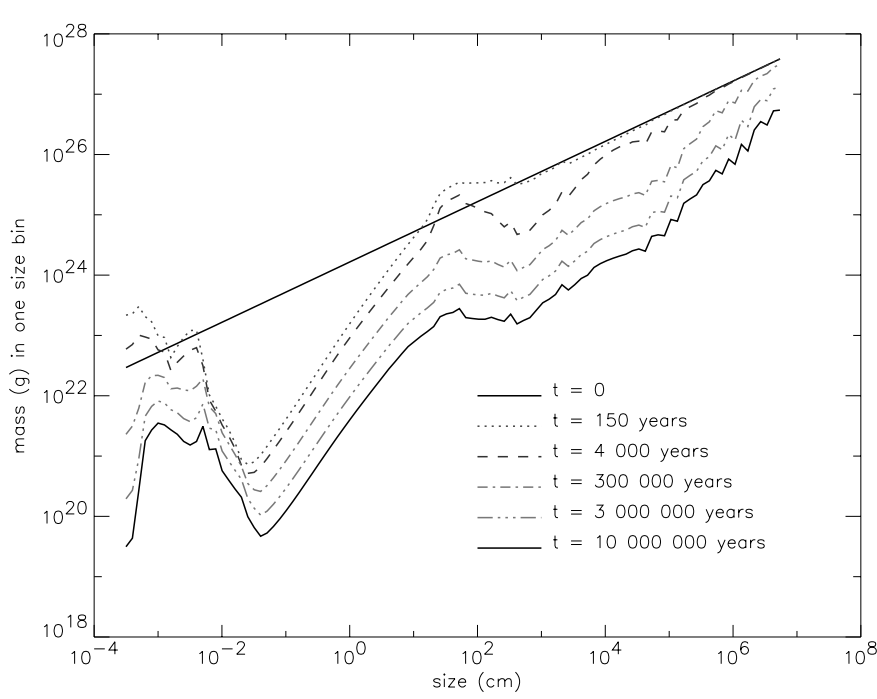

Fig. 4. Size distribution at 5 different epochs for the massive-disc case, where the total mass of the system is chosen in order to match the planetesimal mass estimates deduced from FEB mechanism analysis (see Sect. 2.2).

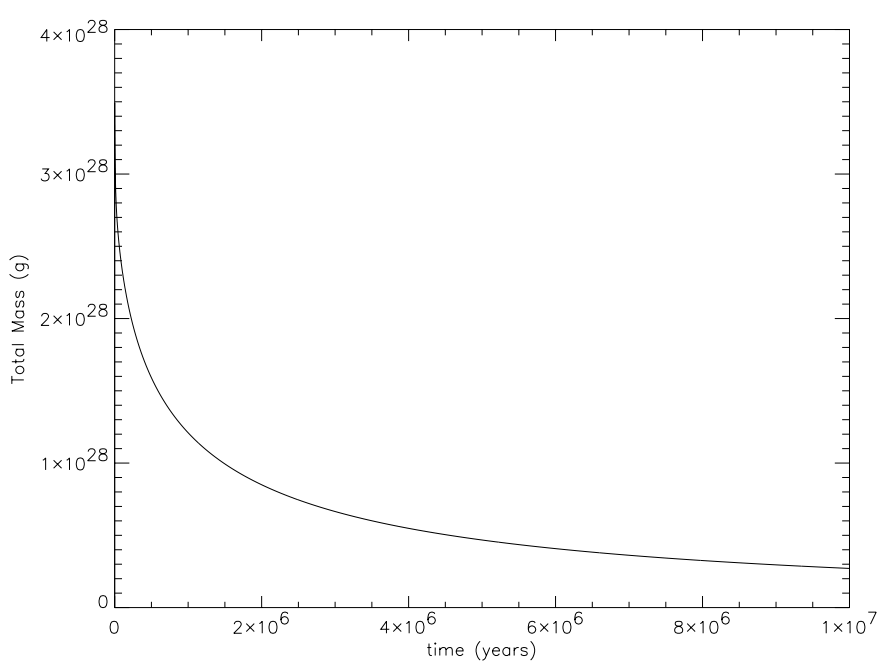

Fig. 5. Temporal evolution of the system's total mass for the massive disc case.

\subsection{Role of the disc's excitation}

Apart from the nominal case with $\langle i\rangle=1 / 2\langle e\rangle=0.05$, two different disc excitations have been tested: one low excitation case at $\langle i\rangle=0.0125=1 / 2\langle e\rangle$ and one high excitation case at $\langle i\rangle=0.1=1 / 2\langle e\rangle$, all other parameters being equal (Fig. 6).

As could logically be expected, the total mass loss is much higher in the high excitation case, $\simeq 18 \%$, than in the low excitation case, $\simeq 4 \%$. Nevertheless, the steady-state regime profile is significantly different for both runs. The density well in the $0.01-1 \mathrm{~cm}$ range is in particular much deeper for the dynamically cold disc. This is a fully logical result when considering the fact that the radiation pressure induced high $e$ of the smallest grains only weakly depends on the dynamical state of the parent bodies (Eq. (14)). As a consequence, the contrast between the excitation, and thus the shattering power, of the smallest grains and that of the rest of the particles is very

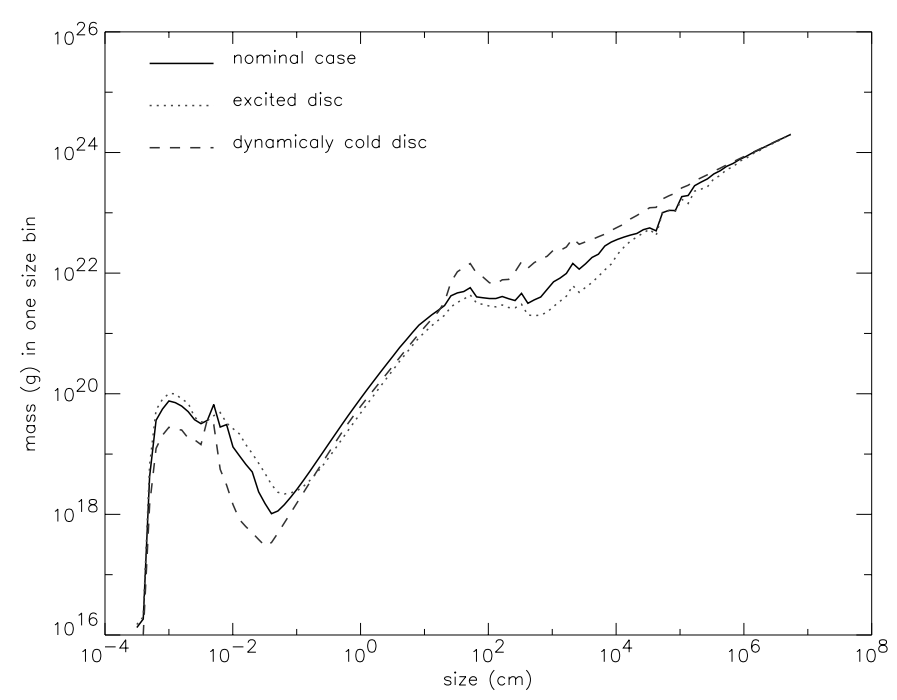

Fig. 6. Final distribution (at $t=10^{7}$ years) for different levels of the disc's dynamical excitation.

high. The destruction rate of bodies in the $0.01-1 \mathrm{~cm}$ range by small grains is thus at the same level than in the nominal case, whereas the production rate of $0.01-1 \mathrm{~cm}$ grains by collisions between bigger objects is much lower, hence the deeper density well. Conversely, for the high excitation case, the contrast between the small grains' and bigger objects' destructive powers is significantly damped, hence a shallower density drop in the $0.01-1 \mathrm{~cm}$ range.

\subsection{Porosity, value of $R_{\mathrm{pr}}$}

As previously discussed, our nominal case corresponds to compact low porosity grains and $R_{\mathrm{pr}}=5 \mu \mathrm{m}$. We investigated different porosities, and thus different $R_{\mathrm{pr}}$ values, all other parameters being equal (with always 2 size boxes below $R_{\mathrm{pr}}$ ). As can be clearly seen in Fig. 7, changing the value of $R_{\mathrm{pr}}$ results mainly in shifting the wavy structure without affecting its overall profile. Although it is not strictly speaking a homothetic shift, mainly because of the complexity of the $Q_{*}$ prescription, differences are minor ones, and the density drop is always located at roughly $100 R_{\mathrm{pr}}$.

\section{5. $Q_{*}$ prescription}

Taking the Housen \& Holsapple (1990) and Holsapple (1994) prescription for $Q_{*}$ leads to a final size distribution which is remarkably close to the nominal Benz \& Asphaug (1999) case (Fig. 8). The main difference is a more defined density drop for objects bigger than $10^{5} \mathrm{~cm}$, which is directly due to the fact that $Q_{*}$ values for bodies in this size range are significantly lower than in the Benz \& Asphaug (1999) model. This deficit in large bodies is the reason for the more significant total mass loss in the system (Fig. 2), since these bodies contain most of the system's mass. Note however that this total mass loss does not reach very large values, remaining limited to less than $30 \%$, and that the global dust to planetesimals mass ratio is also relatively close to the nominal case value (Fig. 3). 


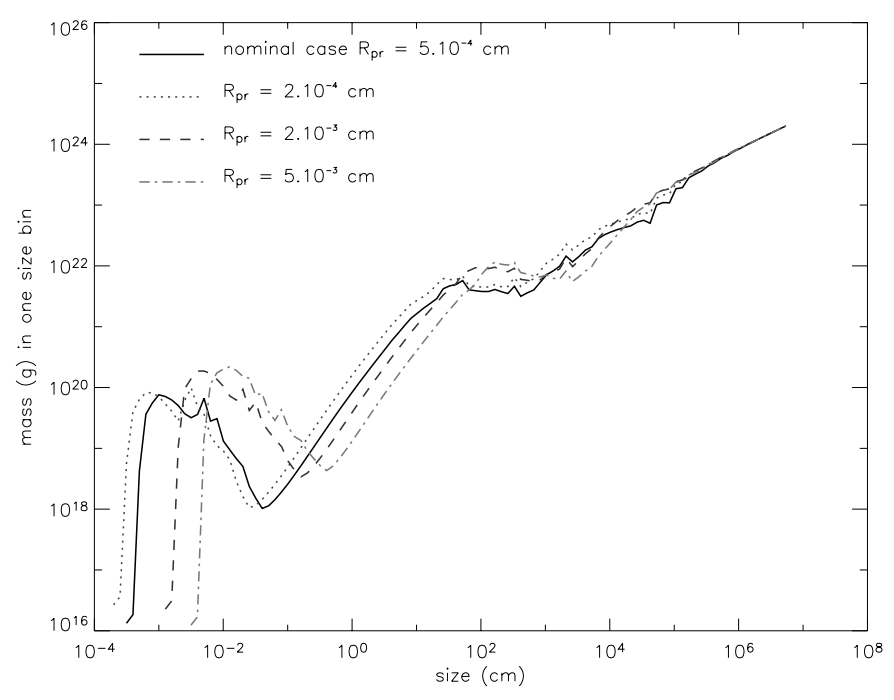

Fig. 7. Final distribution (at $t=10^{7}$ years) for different values of $R_{\mathrm{pr}}$.

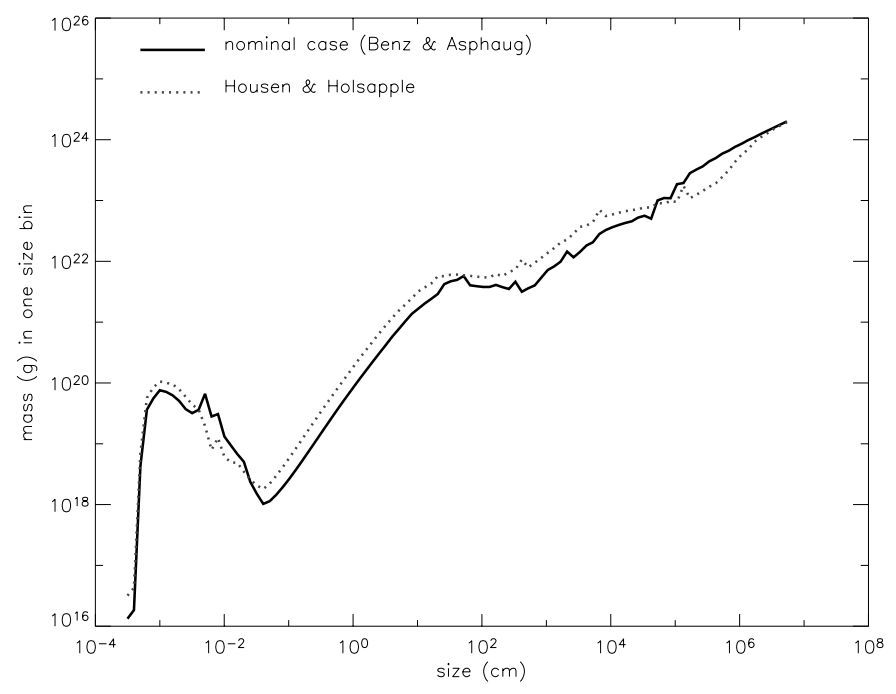

Fig. 8. Final mass distribution (at $t=10^{7}$ years) for different $Q_{*}$ prescriptions.

\subsection{Fragmentation and cratering prescriptions}

As previously shown, the main free parameters for our fragmentation prescription are the ratio $q_{1} / q_{2}$ and the size $R_{\mathrm{s}}$ of the slope transition. These parameters were both explored in independent runs whose results are presented in Fig. 9. As can be clearly seen, the values of $q_{1} / q_{2}$ and $R_{\mathrm{S}}$ only moderately affect the final size distribution within the system.

As appears in Fig. 10, the cratering prescription, in particular the value of the excavation coefficient $\alpha$, has a more significant effect on the physical evolution of the system. Taking a very hard material prescription $\left(\alpha=10^{-9}\right)$ leads indeed to a final size distribution which is very close to a $\mathrm{d} N=C R^{-3.5} \mathrm{~d} R$ power law. The density drop in the sub-centimeter size-range is in particular significantly reduced, with only a factor 8 drop in a narrow region around $10^{-2} \mathrm{~cm}$. Conversely, the very soft material run $\left(\alpha=4 \times 10^{-8}\right)$ leads to a deeper density drop and a more pronounced wavy structure throughout the size distribution. This dependency of the size distribution profile on the
Table 4. This table sums up, for all objects within 3 different size ranges, the respective amount of mass that is removed by all cratering and all fragmenting impacts. These values are obtained in the steady state regime for the nominal case.

\begin{tabular}{lcccc}
\hline \hline Size range $(\mathrm{cm})$ & $R<5 \times 10^{-3}$ & $0.01<R<1$ & $10^{5}<R$ & total \\
\hline Fraction of & & & & \\
mass removed: & & & & \\
by fragmentation & 0.89 & 0.13 & 0.76 & 0.67 \\
by cratering & 0.11 & 0.87 & 0.24 & 0.33 \\
\hline
\end{tabular}

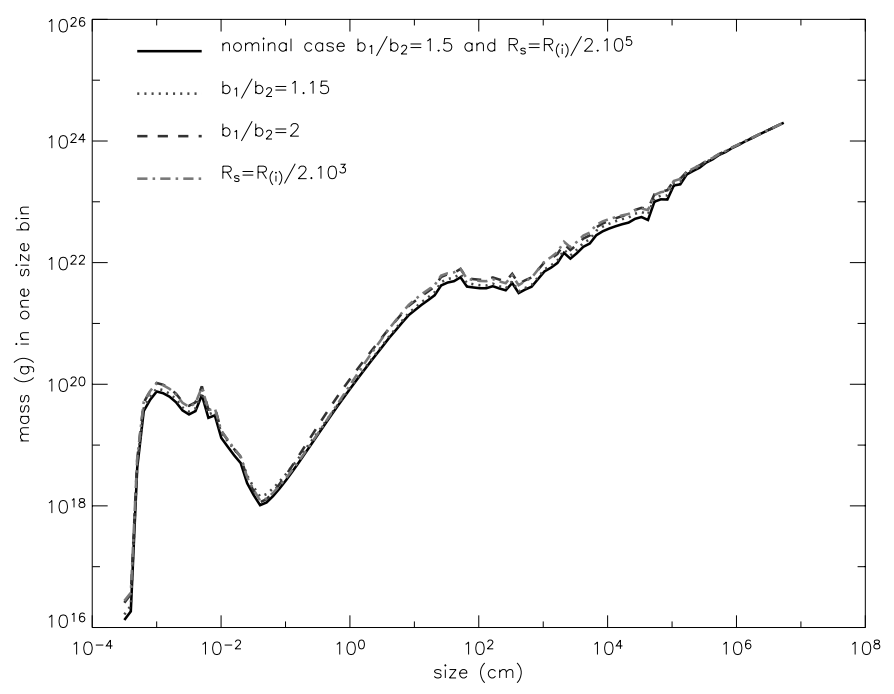

Fig. 9. Final mass distribution (at $t=10^{7}$ years) for different values of the free parameters of our bimodal power law: i.e. the ratio of their slopes $q_{1} / q_{2}$ and the position of the slope changing size $R_{\mathrm{S}}$ with respect to the size $R_{\mathrm{i}}$ of the impacted body.

cratering prescription is easily understandable when realizing that, in the 0.01 to $1 \mathrm{~cm}$ domain, cratering is a much more efficient process than fragmentation in terms of mass removal (Table 4); mainly because of the cratering events due to the high $e$ grains in the $R_{\mathrm{pr}}$ to $\simeq 10 R_{\mathrm{pr}}$ range. Note however, that for the system as a whole, it is fragmentation which is clearly the dominating mass removing process (Table 4).

\subsection{Collisions outside the inner disc}

As discussed in Sect. 3.2, we chose to perform additional test runs checking the possible influence of small particles removal by collisions outside the inner disc. This effect is arbitrarily parameterised by the two parameters $\mathrm{d} t_{c l(k)}$ and $f_{c l(k)}$ (see Sect. 3.2).

We present here results obtained for the most extreme case, where $\mathrm{d} t_{c l(k)}$ was unrealistically supposed to be equal to one orbital period of a $\beta_{k}$ particle. As appears clearly from Fig. 11, differences to the nominal case remain marginal. As expected, the main difference is found for bins just below the $\beta=0.5$ cut-off, with a factor 4 number density difference for the first bin corresponding to bound orbits $\left(\beta_{k}=0.49\right)$. Nevertheless, this difference already drops to $25 \%$ for particles of size $2 R_{\mathrm{pr}}$ $\left(\beta_{k}=0.24\right)$. As a consequence, the sharp density drop induced by the apoastron collisions effect remains confined to a 


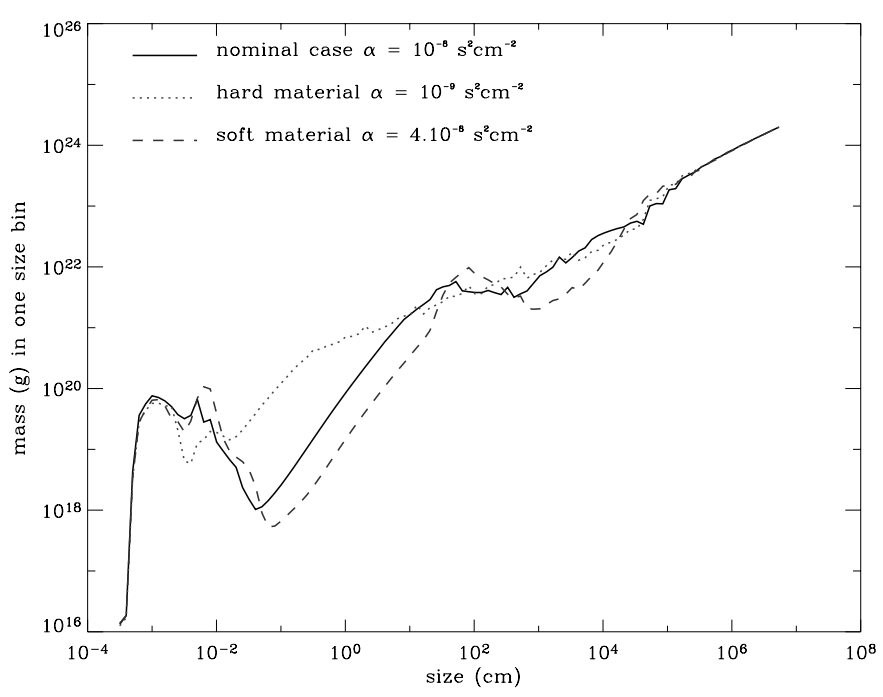

Fig. 10. Final mass distribution (at $t=10^{7}$ years) for different values of the excavation coefficient $\alpha$.

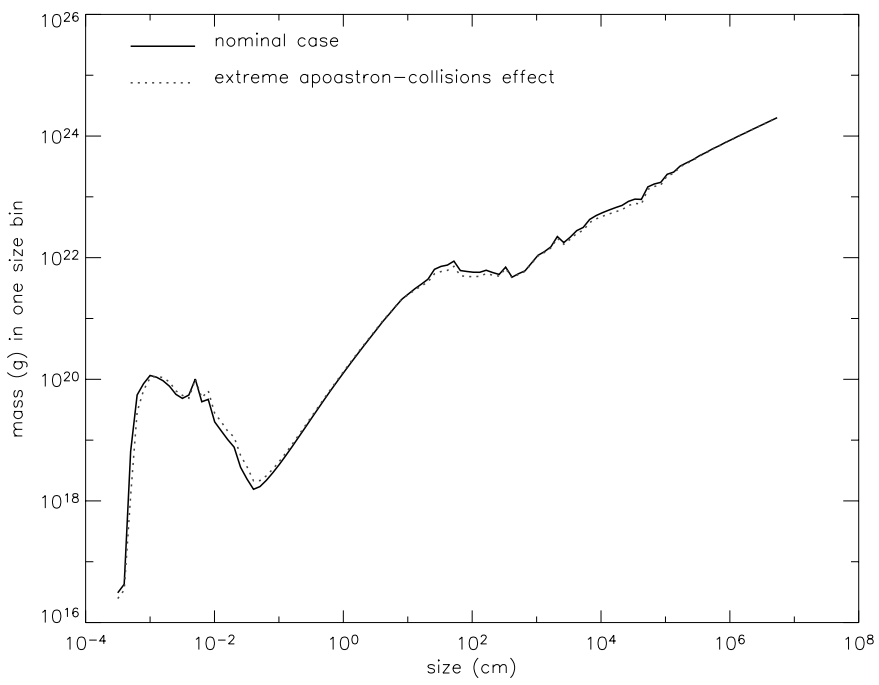

Fig. 11. Final mass distribution (at $t=10^{7}$ years) for an academic case with a very efficient removal of small particles by hypothetic collisions in the $r>10 \mathrm{AU}$ region (see text for details).

narrow size range of particles. Furthermore, this density drop does not have significant consequences on the rest of the size distribution and doesn't affect the global profile of the wavy size distribution. This is because it affects particles which are already strongly depleted because of their high $a$ and $e$ (low $\left.f_{i(k)}\right)$ values. Besides, this removing effect's dependency on $\beta$ is relatively similar to that of the one induced by low $f_{i(k)}$ ) values; it will thus only tend to reinforce an effect already taken into account. Thus, further depleting these populations does not lead to drastic changes.

\section{Discussion}

\subsection{The massive disc case. Problems with the FEB scenario}

One of the most obvious and easily understandable results is the strong mass loss for the massive disc case. As previously stated, the system loses more than $90 \%$ of its total mass over $10^{7}$ years. The problem gets even worse when trying to extrapolate this mass loss to the past. This might be done when noticing that, apart from the initial transition phase, the system's mass loss in the steady state regime might be fitted by a $M_{(t)}=(a+b . t)^{-1}$ law (which is a logical result since the mass loss is proportional to the square of the system's total mass), with $a=5.2 \times 10^{-29} \mathrm{~g}^{-1}$ and $b=3.2 \times 10^{-35} \mathrm{~g}^{-1} \mathrm{~s}^{-1}$. It is easy to see that extrapolating this law to the past leads to masses that become rapidly unrealistically high. Thus, the planetesimal density required to sustain the FEB phenomenon corresponds to a very rapidly evolving system and cannot be maintained over a long period of time. One could argue that considering a dynamically colder system would significantly reduce the system's mass loss. But this would not solve the problem, since the strength of the FEB producing mean-motion resonances directly depends on the system's excitation, so that reducing the disc's excitation would require an even higher number density of planetesimals in order to get the observed FEB rate (Thébault \& Beust 2001).

The problem with the sofar accepted FEB scenario is then the following: from combined observations and modelling, the massive disc required to sustain the observed activity should erode significantly within less than $10^{6}$ yrs, giving a natural end to the FEB phenomenon. If this was to be the case, then we would be presently witnessing a very transient phenomenon. This does not appear satisfactory from a statistical point of view. Should this mean that the FEB scenario should be rejected as a whole? We believe that it is too early to state anything definitely. There are several reasons for that:

We must first recall that the estimate for the necessary disc population for sustaining the FEB activity is derived through a chain calculation which depends on several poorly constrained parameters (see the extended discussion in Thébault \& Beust 2001). Thébault \& Beust (2001) (Eqs. (7) and (8)) showed that the most crucial parameter is here $R_{\mathrm{FEB}}$, i.e. the minimum size of bodies able to become observable FEBs, since the deduced total mass scales roughly as $R_{\mathrm{FEB}}^{q-1}$ in the simplified case where a power law of index $q$ applies for the size distribution above $R_{\mathrm{FEB}}$, so any change to $R_{\mathrm{FEB}}$ may induce drastic changes to the estimated disc mass. Thébault \& Beust (2001) assumed $R_{\text {FEB }}=15 \mathrm{~km}$, but this value is poorly known and could easily vary by one order of magnitude. $R_{\mathrm{FEB}}$ exists because bodies smaller than $R_{\mathrm{FEB}}$ are assumed to evaporate too quickly and consequently make too few periastron passages in the refractory evaporation zone $(\$ 0.4 \mathrm{AU})$ to significantly contribute to the observable spectral activity. The value of $R_{\mathrm{FEB}}$ is thus related to the evaporation rate of the FEBs themselves. Simulations of the dynamics of the material produced by FEB evaporation (Beust et al. 1996) led to derive production rates of a few $10^{7} \mathrm{~kg} \mathrm{~s}^{-1}$ as necessary to yield observable spectral components. We believe that this part of the scenario needs to be revised. The main reason for that is that in Beust et al. (1996) simulations, the material escaped from the FEBs was assumed for simplicity to consist of the metallic ions we study and volatile material. The metallic ions undergo a strong radiation pressure from the star while this is not the case for the volatiles. Hence the volatiles retain the metallic ions around the FEB coma for a 
while, leading to observable components. The production rate was then derived from the necessary amount of volatiles to retain the ions, and from assumptions about the chemical composition of the body. All this is obviously the weakest part of the scenario.

Besides, Karmann et al. (2001) showed that if the FEB progenitors are supposed to originate from 4-5 AU from the star, they should no longer contain ices today (i.e. volatile material), apart from an eventual residual core. More recently, Karmann et al. (2003) made an independent theoretical study of the evaporation behaviour of such objects when they gradually approach the star on repeated periastron passages. The evaporation rates derived are thus independent from any observation. Basically, this work shows that $\sim 10 \mathrm{~km}$ sized bodies fully evaporate with repeated periastron passages, and that evaporation rates of a few $10^{7} \mathrm{~kg} \mathrm{~s}^{-1}$ are actually reached, but this occurs only when the periastron is less than $\sim 0.2 \mathrm{AU}$, i.e. well inside the dust evaporation zone and shortly before the final evaporation of the body. Before that, any FEB entering the dust evaporation zone $(\lessgtr 0.4 \mathrm{AU})$ but for which the periastron has not yet reached $0.2 \mathrm{AU}$ actually evaporates, but at a weaker rate. If it is small, it thus survives more periastron passages than in previous estimates, and may contribute to the observational statistics.

However, whether bodies with no or very few volatiles may generate observable components is questionable, as volatiles have a crucial role in the dynamics of the metallic ions. Within the refractory material, some species suffering low radiation pressure, and that are probably abundant (carbon, silicon, ... ) may play the retaining role of volatiles. Obviously this question must be reinvestigated with more realistic simulations, but a probably outcome will be that $R_{\mathrm{FEB}}$ could end up to be at least one order of magnitude less than previously estimated. In this context, our chain calculation would lead to a much lower disc mass necessary for sustaining the FEB activity.

In this context, it is impossible to rule out the FEB scenario on this basis alone, but this remain a problematic possibility. All we can presently state is that the disc populations inferred by Thébault \& Beust (2001) are unrealistic and that the FEB scenario should at least be reinvestigated much more carefully.

\subsection{Departure from the $R^{-3.5}$ profile}

Putting aside the peculiar massive disc problem, the most striking result, present for almost all tested simulations, is a final size-distribution that significantly departs from a $R^{-3.5}$ power law, especially in the small size domain. The only exception to this behaviour is a run with a very hard material parameter for the cratering prescription, which means that alternative sizedistribution profiles cannot be completely ruled out, although they seem to represent a marginal possibility. Of course, due to the complexity of the studied problem, all free parameters could not be exhaustively explored. Besides, there are some parameters that are strongly coupled, i.e. fragmentation and cratering prescriptions should in principle not be independently explored. Nevertheless, there seems to be a global tendency towards a common feature which consists of a lack of objects close to the limiting ejection size $R_{\mathrm{pr}}$, a density peak at $\simeq 2 R_{\mathrm{pr}}$ and a sharp density drop compared to the $R^{-3.5}$ law, of one or two orders of magnitude in mass, at $\simeq 100 R_{\mathrm{pr}}$. It is also important to note that a very badly constrained parameter such as the disc's dynamical excitation does not seem to have a crucial influence on the profile, thus reinforcing the genericity of this result.

These departures from the $\mathrm{d} N=C R^{-3.5} \mathrm{~d} R$ power law do not lead to radical changes in the global dust vs. planetesimal mass ratio in the system, which only decreases by a factor $\simeq 3-4$. If $2 \times 10^{22} \mathrm{~g}$ is a typical value for the amount of dust (i.e. $R<1 \mathrm{~mm}$ ) in the inner $10 \mathrm{AU}$ region (see Sect. 1), then we estimate from our results that the corresponding mass of $1 \mathrm{~km}<R<50 \mathrm{~km}$ objects should be $\simeq 3.5-7 \times 10^{-2} M_{\oplus}$, which remains a value comparable to the one roughly derived from a $R^{-3.5}$ power law (see Sect. 1). Even stretching this value up to the $1 \mathrm{~km}<R<500 \mathrm{~km}$ range does not lead to more than $0.15 M_{\oplus}$ of "large" objects. Our calculations thus quantitatively confirm what had been previously inferred from questionable assumptions (an $R^{-3.5}$ power law): there $i s$ a lack of objects, that holds even for large planetesimals, in the inner disc.

This is an additional problem for the FEB scenario, since this value is far from being enough in order to account for the sharp incompatibility between the amount of observed dust and the required amount of FEB inducing planetesimals. In any case, it appears clearly that the FEB model as it is currently accepted cannot be compatible with a "reasonable" estimate of the dust production rate in the inner disc.

\subsection{Collisional erosion vs. cometary evaporation}

Let us recall that the precise SED fit performed by Li \& Greenberg (1998) was obtained assuming that the dust is of pure cometary origin and is not affected by collision processes. On the contrary, in our simulations we implicitly made the assumption that the inner $\beta$ Pictoris dust disc is made of collisional debris. We do believe that our results retrospectively justify this assumption, although without ruling out the possible presence of evaporating bodies, and this for several reasons.

1. For what is presently known about the inner disc, the collision production hypothesis seems to be quantitatively more generic than the concurrent cometary evaporation. In his Eq. (2) Lecavelier (1998) proposed a simple expression in order to estimate the number $N_{\text {co }}$ of currently evaporating comets in a dust disc, where $N_{\text {co }}$ directly depends on $M_{\text {dust }}$, the total dust mass, and $t_{\text {dust }}^{-1}$, the typical lifetime for a dust particle before destruction. Taking the same Halley-at-1AU evaporation rates as Lecavelier (1998) and bodies of $20 \mathrm{~km}$ in radius leads to $N_{\mathrm{co}} \simeq 1.5 \times 10^{4} *\left(t_{\text {dust }} / 10^{4} \mathrm{yrs}\right)^{-1}$. Considering that collisional lifetimes of dust grains are of the order of $10^{3}$ years in the inner Beta-Pic disc (Fig. 12), we get $\simeq 2.6 \times 10^{-3} M_{\oplus}$ of evaporating comets in the $r<$ $10 \mathrm{AU}$ region. This value represents $\simeq 10 \%$ of the estimated total mass of kilometre-sized objects (cf. Table 1), which should mean that one out of ten planetesimal objects is an evaporating comet. Although such a possibility cannot be completely ruled out, it seems nevertheless rather unlikely 


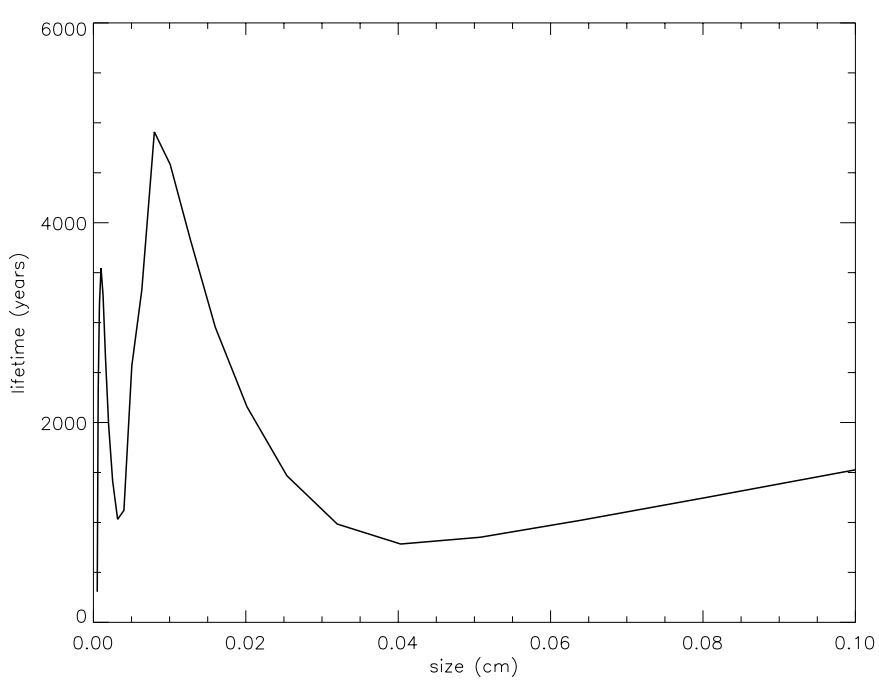

Fig. 12. Typical lifetime, as a function of its size, of a dust particle in the inner disc before destruction by collision (steady state regime of the nominal case).

since Karmann et al. (2001) has showed that all kilometresized objects originating from the $5 \mathrm{AU}$ region should no longer contain volatile material today. One could argue that the previous calculation depends on poorly constrained parameters and that the requested number of evaporating bodies could be lower. But even in this case there is one crucial problem to solve: what is the mechanism constantly refilling the inner disc with so many fresh comets? The main difficulty is that this refilling has to be very effective and rapid, since the average time needed for a $10 \mathrm{~km}$ body at 5 AU to lose all its volatile material is less than 100 years (see Fig. 2 of Karmann et al. 2001).

2. A more conclusive argument is that the present simulations show that the presence of $\simeq 2 \times 10^{22} \mathrm{~g}$ of dust in the $<10 \mathrm{AU}$ region might be explained, within a moderate mass disc, by collisional processes alone. Moreover, such a low mass disc is consistent with what should be expected, in the inner disc, considering the age of the system: a disc of debris left over after the accretion process of planetary embryos (see next subsection). In short, there is no need for a cometary activity in terms of production of the observed dust.

3. In any case, even if all the dust was to be produced by evaporating comets, then the present simulations show that mutual collisions within such $\mathrm{a} \simeq 2 \times 10^{22} \mathrm{~g}$ dust disc would anyway be unavoidable. This would strongly affect the size distribution, which would probably tend towards the steady-state profiles displayed in Sect. 4.

Of course, these arguments are relevant only for the inner $\beta$ Pic disc. We do not rule out the possibility that comet evaporation could be a dominant dust-production source in the outer parts of the system, as suggested by the Orbital Evaporating Bodies scenario proposed by Lecavelier (1998) for the region beyond $70 \mathrm{AU}$. This might appear to be a somewhat paradoxal result, since evaporation processes should be more effective in the inner regions. But let us once again stress that these higher evaporation rates are precisely what makes it difficult to find a way to sustain evaporation activity over long time scales in the inner disc (as previously mentioned, a $10 \mathrm{~km}$ object evaporates in less than 100 years at $5 \mathrm{AU}$ ). At larger distances, volatile evaporation rates are much lower, thus reducing the crucial problem of "refilling" the evaporation region with fresh material. Of course, distances from the star must remain within the limiting distance at which evaporation is possible, i.e. 100-150 AU for CO (Lecavelier et al. 1996).

\subsection{Presence of already formed planetary embryos}

Of course, one cannot rule out the possible presence of isolated much more massive objects, such as planets or planetary embryos, whose isolation decouples them from the collisional cascade responsible for the dust production (a possibility considered by Wyatt \& Dent (2002) for the Fomalhaut system). In fact, the low mass in the dust-to-planetesimals range could be interpreted as the consequence of the presence of such planetary embryos: most of the initial mass of the system would already have been accreted in these embryos, leaving a sparse disc of remnants. This would be in accordance with the estimated age of the system, a few $10^{7}$ years, which significantly exceeds the expected timespan for the formation of planetary embryos (e.g. Lissauer 1993), so that if embryos have to form, then they should be already here.

Another argument previously proposed in favour of the presence of already formed massive embryos is that such objects are a good way to explain the disc's thickness. Artymowicz (1997) estimated that numerous Moon-sized bodies are required in order to induce vertical velocity dispersions of smaller bodies of the order of $0.1 v_{\text {kep }}$. Nevertheless, as pointed out by Mouillet et al. (1997), a giant planet on a slightly inclined orbit (like the planet required to explain the warp in the outer regions) could achieve just the same result: rapid precession of the dust particles orbits in the inner regions would lead to thicken the disc so that the aspect ratio appears to be equal to the planet's inclination.

\section{Conclusions and perspectives: Towards a coherent picture of the inner $\beta$ Pictoris disc?}

The present study show that the observed $10^{21}$ to $10^{22} \mathrm{~g}$ of dust in the inner disc is compatible with what would be expected from a collisional cascade within a disc of a few $10^{-2} M_{\oplus}$ bodies ranging from micron to kilometre-sized objects, without the need for any additional cometary-evaporation activity. And even if there was such a cometary activity, the required density of objects would inevitably lead to important collisional effects.

Simulations also show that the size distribution settles towards a quasi-equilibrium state that strongly departs from the classical $\mathrm{d} N \propto R^{-3.5} \mathrm{~d} R$ Dohnanyi power law. This is particularly true for the smallest grains close to the radiation pressure ejection limit. However, these departures do not too strongly affect the global Dust-to-Planetesimal mass ratio and cannot account for the incompatibility between the small amount of observed dust and the huge number of kilometre-sized FEBs requested to sustain the transient absorption features activity. Furthermore, our runs show that this requested mass of FEBs 
leads to a much too rapidly collisionaly eroding disc that cannot survive on long timescales. The FEB scenario thus cannot hold in its present form and has to be seriously revised.

We might thus converge towards a coherent picture of the inner $\beta$ Pictoris disc: this inner disc should be boundered by one giant planet of $\simeq 1 M_{\text {Jup }}$, located around $10 \mathrm{AU}$ on a slightly inclined (in order to explain the observed outer warp) and possibly eccentric orbit (in order to trigger the FEB activity). The observed amount of dust should be produced by collisional erosion within a low mass disc. Such a low mass disc could be made of debris leftover after the accretion of one or several planetary embryos, the presence of which is fully compatible with the estimated age of the system, i.e. a few $10^{7}$ years. In other words, we should be now witnessing a planetary system in a late or at least intermediate stage. The bulk of the accretion process is over, but a consequent disc of remnants is still present and collisionaly eroding.

Our results would also help putting new constraints on the SED fits that are usually performed to derive dust densities and radial distributions from observed spectra. Let us recall that the dust mass estimations for the inner disc, which we used as inputs for our simulations, have been computed either by postulating that grains are of cometary origin (Li \& Greenberg 1998) or by doing a pure mathematical fit with several free parameters (Pantin et al. 1997). In this respect, it would be interesting to perform a work similar to that of Li \& Greenberg (1998) but with a population of collisionaly produced grains as input. An interesting attempt at doing such a kind of study has been recently made by Wyatt \& Dent (2002) in their very detailed study of the Fomalhaut's debris disc. Nevertheless, their precise fit of the SED was made assuming a single power law for the size distribution (even though the authors were fully aware of the fact that such an academic distribution cannot hold for the smallest grains because of the cutoff effect). Such an SED-fit analysis goes beyond the scope of the present paper and requires additional work.

It requires in particular to model the whole $\beta$ Pictoris disc and not only the innermost parts that only partially contribute to the total flux. Only then could the obtained size distribution be compared to SEDs integrated over the whole disc. A crucial problem would probably be to see if the underabundance of millimetre-sized objects that we obtained in the inner disc is also to be found for the system as a whole; this would then contradict previous estimates stating that the observed mass of millimetre objects is in accordance with a -3.5 equilibrium power law (Artymowicz 1997). Such a study should of course also address more deeply the question of the physical nature of the dust grains. It will be the purpose of a forthcoming paper.

Acknowledgements. The authors wish to thank M. Wyatt and P. Artymowicz for fruitful comments and discussions. J. C. Augereau was supported by a CNES grant and a European Research Training Network "The Origin of Planetary Systems" (PLANETS, contract number HPRN-CT-2002-00308) fellowship.

\section{References}

Augereau, J. C., Lagrange, A. M., Mouillet, D., Papaloizou, J. C. B., \& Grorod, P. A. 1999, A\&A, 348, 557
Augereau, J. C., Nelson, R. P., Lagrange, A. M., Papaloizou, J. C. B., \& Mouillet, D. 2001, A\&A, 370, 447

Artymowicz, P. 1997, Ann. Rev. Earth Planet. Sci., 25, 175

Barrado y Navascués, D., Stauffer, J. R., Song, I., \& Caillault, J.-P. 1999, ApJ, 520, L123

Benz, W., \& Asphaug, E. 1999, Icarus, 142, 5

Beust, H., Lagrange, A.-M., Plazy, F., \& Mouillet, D. 1996, A\&A, 310,181

Beust, H., \& Morbidelli, A. 2000, Icarus, 143, 170

Beust, H., Karmann, C., \& Lagrange, A.-M. 2001, A\&A, 366, 945

Boggess, A., Bruhweiler, F. C., Grady, C. A., et al. 1991, ApJ, 377, L49

Campo Bagatin, A., Cellino, A., Davis, D., Farinella, P., \& Paolicchi 1994, Planet. Space Sci., 42, 1079

Chini, R., Kruegel, E., Kreysa, E., Shustov, B., \& Tutukov, A. 1991, A\&A, 252, 220

Davis, D., \& Ryan, E. 1990, Icarus, 83, 156

Dohnanyi, J. S. 1969, JGR, 74, 2531

Fujiwara, A., Kamimoto, G., \& Tsukamoto, A. 1977, Icarus, 31, 277

Fujiwara, A., Cerroni, P., Davis, D., Ryan, E., \& di Martino, M. 1989, in Asteroids II, ed. R. P. Binzel, T. Gehrels, \& M. S. Matthews (Tucson: Univ. of Arizona Press), 240

Holland, W. S., Greaves, J. S., Zuckerman, B., et al. 1998, Nature, 392,788

Holsapple, K. 1994, Planet. Space Sci., 42, 1067

Housen, K., \& Holsapple, K. 1990, Icarus, 84, 226

Kalas, P., \& Jewitt, D. 1995, AJ, 110, 794

Kalas, P., Larwood, J., Smith, B. A., \& Schultz, A. 2000, ApJ, 530, L133

Karmann, C., Beust, H., \& Klinger, J. 2001, A\&A, 372, 616

Karmann, C., Beust, H., \& Klinger, J. 2003, A\&A, submitted

Knacke, R. F., Fajardo-Acosta, S. B., Telesco, C. M., et al. 1993, ApJ, 418,440

Lagage, P. O., \& Pantin, E. 1994, Nature, 369, 628

Lagrange, A.-M., Backman, D. E., \& Artymowicz, P. 2000, Protostars and Planets IV, 639

Larwood, J. D., \& Kalas, P. G. 2001, MNRAS, 323, 402

Lecavelier des Etangs, A. 1998, A\&A, 337, 501

Lecavelier des Etangs, A., Deleuil, M., Vidal-Madjar, A., et al. 1995, A\&A, 299, 557

Lecavelier des Etangs, A., Vidal-Madjar, A., \& Ferlet, R. 1996, A\&A, 307,542

Li, A., \& Greenberg, M. 1998, A\&A, 331, 291

Lissauer, J. 1993, ARA\&A, 31, 129

Lissauer, J., \& Stewart, G. 1993, in Protostars and Planets III (Tucson: Univ. of Arizona Press), 1061

Mouillet, D., Larwood, J. D., Papaloizou, J. C. B., \& Lagrange, A.-M. 1997, MNRAS, 292, 896

Pantin, E., Lagage, P. O., \& Artymowicz, P. 1997, A\&A, 327, 1123

Petit, J.-M., \& Farinella, P. 1993, Celest. Mech. Dynam. Astron., 57,1

Smith, B., \& Terrile, R. 1984, Science, 226, 1421

Stern, A., \& Colwell, J. 1997, ApJ, 490, 879

Tanga, P., Cellino, A., Michel, P., et al. 1999, Icarus, 141, 65

Thébault, P., \& Beust, H. 2001, A\&A, 376, 621

Thébault P., Marzari, F., \& Scholl, H. 2002, A\&A, 384, 594

Vidal-Madjar A., Lagrange-Henri, A.-M., Feldman, P. D., et al. 1994, A\&A, 290, 245

Wahhaj, Z., Koerner, D. W., Ressler, M. E., et al. 2003, ApJ, 584, L27

Wyatt, M. C., \& Dent, W. R. F. 2002, NNRAS, 334, 589

Zuckerman, B., \& Becklin, E. E. 1993, ApJ, 414, 793 